\title{
Editorial
}

\section{Introduction to the special issue on the 2011 INFORMS Revenue Management and Pricing Section Conference}

Journal of Revenue and Pricing Management (2012) 11, 567-568. doi:10.1057/rpm.2012.42

This special issue of the Journal of Revenue and Pricing Management contains papers from the 11th Annual INFORMS Revenue Management and Pricing Section Conference, which was held in New York on 23-24 June 2011 at Columbia University. The conference, which featured three parallel tracks and over 80 presentations, was chaired by Guillermo Gallego and Robert Phillips with a program committee of Omar Besbes, Garud Iyengar, Soulaymane Kachani and Garrett van Ryzin. Plenary talks were delivered by Asu Ozdaglar of MIT and Shmuel Oren of University of California at Berkeley. In addition, the Revenue Management and Pricing Section Practice Prize was awarded to a team from Wyndham Exchange and Rentals. The 13th annual section conference will be hosted in Atlanta by Georgia Tech during summer 2013.

The 2011 conference featured talks on a wide array of topics, and this breadth is reflected in the papers in this special issue.

A significant portion of merchandise sales result in product returns. Liberal return policies may increase returns, and they may also stimulate sales. On the other hand, strict return policies may decrease returns and sales. Consequently, return policies offer another tool - besides pricewith which to manage inventory levels. In view of this, retailers may wish to dynamically manage return policies much as they do their pricing policies. In the research paper 'Optimal dynamic return management of fixed inventories', Mehmet Sekip Altug considers a retailer's problem of managing both the return policy and prices when selling a fixed inventory of a good over a finite time horizon with the objective of maximizing expected revenues. The paper describes various structural properties of optimal return management policies, and its results suggest that the approach of dynamically managing returns has promise.

The practice paper 'Fluid approximation to pricing batched products with no product type mixing within batches' by M. Delorme, B. Jenkins, T. Liptrot and D. McCaffrey addresses a problem motivated by situations in which fuel wholesalers deliver oil products to customers. The paper presents a model that can be used to address issues faced by a fuel wholesaler with a fixed number of tanker trucks, which can be used to deliver different grades of fuel. Different fuel grades cannot be delivered simultaneously on the same truck. The main issue is how to find revenue-maximizing prices for the different grades of fuel, taking into account the constraint that fuel grades cannot be mixed together in the delivery tanker trucks. The authors consider a fluid approximation to the original problem and identify properties of an optimal solution. The properties allow the authors to develop a method to solve the fluid approximation that involves considering only a small subset of the possible assignments of fuel grades to delivery trucks. 
Pricing is today an area of emphasis for many companies that engage in business-to-business sales. In 'An exploratory analysis of B2B price changes', Wedad Elmaghraby, Wolfgang Jank, Itir Z. Karaesmen and Shu Zhang study a large data set of sales transactions from a major US grocery products distributor with the aim of understanding $\mathrm{B} 2 \mathrm{~B}$ price changes. They consider a variety of variables that may help explain the likelihood as well as the magnitude of price changes. Their statistical analysis reveals the likelihood of a price change is best explained by the presence of a cost increase or cost decrease and that the effect of a cost increase on the probability is greater than that of a cost decrease. They also find that the amount by which a price changes is best explained by the centralized price change recommended to the salesrep who makes the sale, the actual change in cost and the sign of cost change. Overall, the results shed light on the behavior of salesreps, who exercise considerable power in the $\mathrm{B} 2 \mathrm{~B}$ pricing process.

In the timeshare industry, customers own weeks at particular vacation properties. These customers may sometimes wish to trade those weeks for time at other properties. RCI, a subsidiary of Wyndham Exchange and Rentals, operates an exchange wherein such trades can be made. The exchange works by allowing customers to deposit their weeks. Such deposits are assigned values. Customers may accumulate value from multiple deposits. The accumulated value can then be used to 'purchase' weeks deposited by other customers. An important issue is to determine how much accumulated value should be required to make such a purchase. In the practice paper 'Optimizing vacation exchange', Srinidhi Melkote, Dasong Cao, Preeti Modgil, Sneha Thakkar and Ryan Connelly (all from Wyndham) address this question. Their approach involves a novel mathematical programming formulation and is founded on revenue management principles. The work described in this paper was awarded the 2011 INFORMS Revenue Management and Pricing Section Practice Prize.

Successful markdown pricing is crucial for profitability in settings where major portions of items are sold on markdown. One such setting can be found in fashion retailing. In 'Markdown optimization with an inventory-depletion effect', Andrew Vakhutinsky, Alex Kushkuley and Manish Gupte develop a model to help find monotonically decreasing markdown prices to maximize sales revenue. A key feature of their model is that it allows the demand rate to depend upon the inventory level. This is particularly important when demand is modeled at an aggregate level. An example of such aggregate demand modeling occurs where multiple colors or sizes of a fashion good are considered as a single item. In this case, when all else is equal, the demand rate for the aggregate good (that is, the single item) is generally increasing in its inventory level, because some consumers will not be able to find their size or preferred color at lower inventory levels. Numerical experiments indicate that the authors' approach quickly produces markdown pricing policies that perform very well.

In the addition, this issue contains a futures article by Damian Lord and Ian Yeoman titled 'Pricing Behaviour in Holiday Purchasing Decisions'; the research paper 'Incorporating cancel and rebook behavior in revenue management optimization' by Thomas Gorin, Darius Walczak, Philipp Bartke and Martin Friedemann; and the research paper 'Price bundling in competitive markets' by John Aloysius, Cary Deck and Amy Farmer.

Finally, I thank all of the referees who were involved in the editorial review process for this special issue.

William L. Cooper Department of Industrial and Systems Engineering, University of Minnesota,

Minnesota, USA E-mail: billcoop@umn.edu 